\title{
Core knowledge of geometry can develop independently of visual experience
}

\author{
Benedetta Heimler $^{\mathrm{a}, \mathrm{b}, \mathrm{h}, *, 1}$, Tomer Behor ${ }^{\mathrm{c}, 1}$, Stanislas Dehaene ${ }^{\mathrm{d}, \mathrm{e}}$, Véronique Izard $^{\mathrm{f}, \mathrm{g}}$, \\ Amir Amedi ${ }^{\text {a,b,c }}$ \\ ${ }^{a}$ Department of Medical Neurobiology, Hebrew University of Jerusalem, Hadassah Ein-Kerem, Jerusalem, Israel \\ ${ }^{\mathrm{b}}$ The Baruch Ivcher Institute For Brain, Cognition \& Technology, The Baruch Ivcher School of Psychology, Interdisciplinary Center (IDC), Herzeliya, Israel \\ ${ }^{\mathrm{c}}$ The Cognitive Science Program, The Hebrew University of Jerusalem, Jerusalem, Israel \\ ${ }^{\mathrm{d}}$ Cognitive Neuroimaging Unit, CEA DSV/I2BM, INSERM, Université Paris-Sud, Université Paris-Saclay, NeuroSpin Center, 91191 Gif/Yvette, France \\ ${ }^{\mathrm{e}}$ Collège de France, 11 Place Marcelin Berthelot, 75005 Paris, France \\ ${ }^{\mathrm{f}}$ Integrative Neuroscience and Cognition Center, Université de Paris, 45 rue des Saints-Pères, 75006 Paris, France \\ ${ }^{\mathrm{g}}$ CNRS UMR 8002, 45 rue des Saints-Pères, 75006 Paris, France \\ ${ }^{\mathrm{h}}$ Center of Advanced Technologies in Rehabilitation (CATR), Sheba Medical Center, Tel Hashomer, Israel
}

\section{A R T I C L E I N F O}

\section{Keywords:}

Blindness

Geometry

Geometric selection

Core-knowledge

Spatial reasoning

Spatial exploration

\begin{abstract}
A B S T R A C T
Geometrical intuitions spontaneously drive visuo-spatial reasoning in human adults, children and animals. Is their emergence intrinsically linked to visual experience, or does it reflect a core property of cognition shared across sensory modalities? To address this question, we tested the sensitivity of blind-from-birth adults to geometrical-invariants using a haptic deviant-figure detection task. Blind participants spontaneously used many geometric concepts such as parallelism, right angles and geometrical shapes to detect intruders in haptic displays, but experienced difficulties with symmetry and complex spatial transformations. Across items, their performance was highly correlated with that of sighted adults performing the same task in touch (blindfolded) and in vision, as well as with the performances of uneducated preschoolers and Amazonian adults. Our results support the existence of an amodal core-system of geometry that arises independently of visual experience. However, performance at selecting geometric intruders was generally higher in the visual compared to the haptic modality, suggesting that sensory-specific spatial experience may play a role in refining the properties of this core-system of geometry.
\end{abstract}

"In geometry, what is essential is invisible to the eye: it is only with the mind that one can see rightly" (Emmanuel Giroux, blind mathematician).

Philosophers have debated the emergence of geometric knowledge in human cognition for centuries (Descartes, 1886;Kant, 1998;Plato, 2005): is one born a geometric thinker or is such a skill a result of formal education? This debate continues also in our times. For example, many studies consistently documented that geometric properties such as parallelism, collinearity, or curvilinearity play a crucial role in visual perception, because these properties deffne special "non-accidental" configurations that drive visual perceptual and spatial grouping (Amir, Biederman, \& Hayworth, 2012; Feldman, 1997, 2007; J.Kubilius, Sleurs, \& Wagemans, 2017;Todd, Weismantel, \& Kallie, 2014;Van Gool, Moons, Pauwels, \& Wagemans, 1994;Wagemans, Lamote, \& Van Gool, 1997). Beyond perceptual mechanisms, several studies demonstrated that intuitions about geometry can arise in humans in the absence of formal education, and some of them are even shared with non-human species (Amir, Biederman, Herald, Shah, \& Mintz, 2014;Biederman, Yue, \& Davidoff, 2009; Cheng \& Newcombe, 2005; Dehaene, Izard, Pica, \& Spelke, 2006;Dillon, Huang, \& Spelke, 2013; Gallistel, 1990;Gibson, Lazareva, Gosselin, Schyns, \& Wasserman, 2007; Izard, Pica, Dehaene, Hinchey, \& Spelke, 2011;Izard \& Spelke, 2009; Kayaert \& Wagemans, 2010;Lourenco \& Huttenlocher, 2008; Spelke \& Lee, 2012). By using a visual deviant-ffgure detection task, some of these studies showed that children and adults spontaneously detect a wide range of geometric properties in visual displays (Fig. 1B): for example the topological properties of closure and continuity, or the Euclidean properties of distance, alignment, or parallelism (Dehaene et al., 2006;Spelke, Lee, \& Izard, 2010). Crucially, this was observed in two populations completely nave to

\footnotetext{
* Corresponding author at: Department of Medical Neurobiology, Hebrew University of Jerusalem, Hadassah Ein-Kerem, Jerusalem, Israel.

E-mail address: Benedetta.Heimler@mail.huji.ac.il (B. Heimler).

1 Equal contributions
} 
formal geometry, being devoid of geometric education, namely an Amazonian group, the Mundurucu (Dehaene et al., 2006), and western preschool children (Izard \& Spelke, 2009). Speciffcally, although both Mundurucu and western pre-school children detected geometric cues overall in fewer trials than sighted educated adults, the performance of all three populations were highly correlated across trials. This means that, independently of the group, all participants successfully detected or failed to detect the geometric intruders in the same trials. Thus, these ffndings suggest that basic geometric concepts are built upon core intuitions of spatial reasoning that drive spatial exploration behavior and shape perception, independently of formal education.
However, the role of visual experience in the development of this coresystem of geometry remains unclear. In his celebrated "Letter on the blind", the French enlightenment philosopher Denis Diderot already used the example of Nicolas Saunderson, a first-rate mathematician who was blind since the age of one, to argue in favor of the abstractness and amodal (i.e., sensory-independent) character of mathematical knowledge (Diderot, 1749). Initial empirical evidence supporting Diderot's claim came from a seminal case study by Landau et al. which showed that a 21/2-year-old blind child spontaneously used angular relationships between objects while navigating (Landau, Gleitman, \& Spelke, 1981).
A.
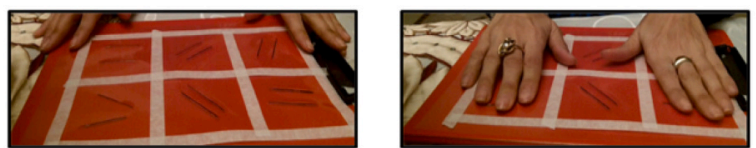

"Explore all the shapes and pick the one you think is the most different one "

\section{Example of a haptic trial (parallel lines trial)}

B. All Experimental Trials and Performance

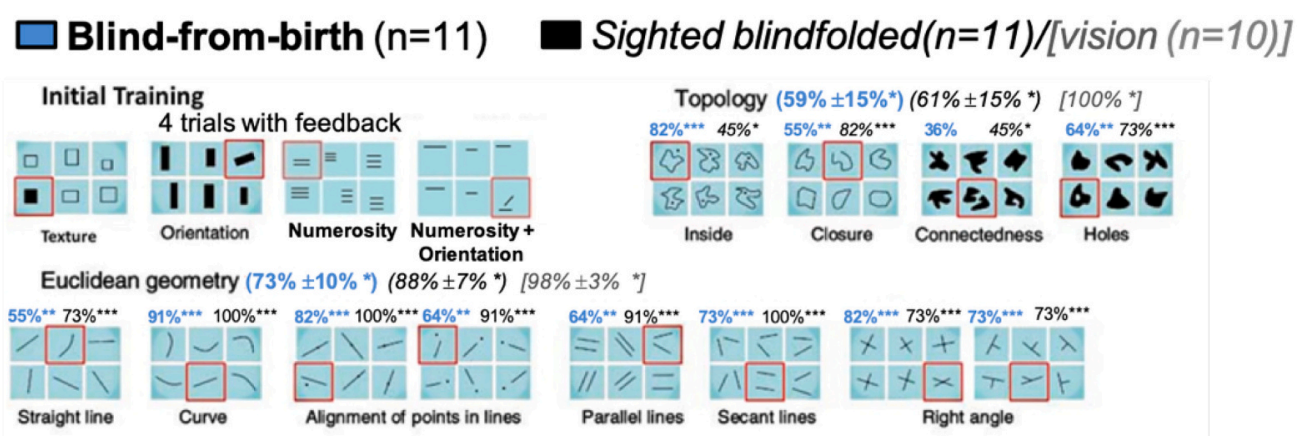

Geometrical figures $(60 \% \pm 10 \%$ *) $(45 \% \pm 10 \%$ *) $[96 \% \pm 5 \%$ *]

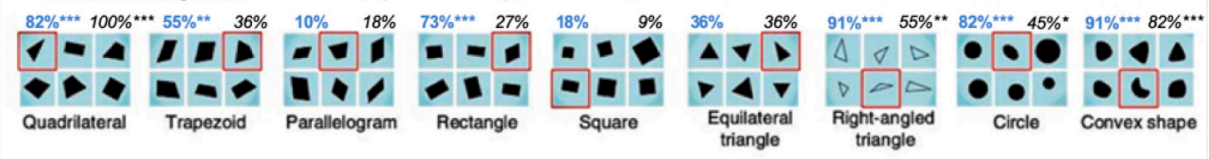

Metric properties $\left(44 \% \pm 11 \%{ }^{*}\right)(51 \% \pm 11 \%$ *) $[82 \% \pm 11 \%$ *]
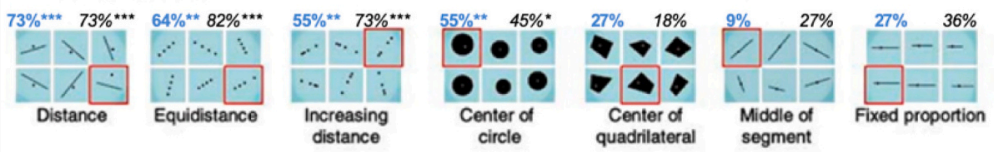

Chiral figures $\left(50 \% \pm 12 \%{ }^{*}\right)\left(68 \% \pm 14 \%{ }^{*}\right)\left[75 \% \pm 13 \%{ }^{*}\right]$

Symmetrical figures $(27 \% \pm 15 \%)(27 \pm 16 \%)\left[79 \% \pm 14 \%{ }^{*}\right]$
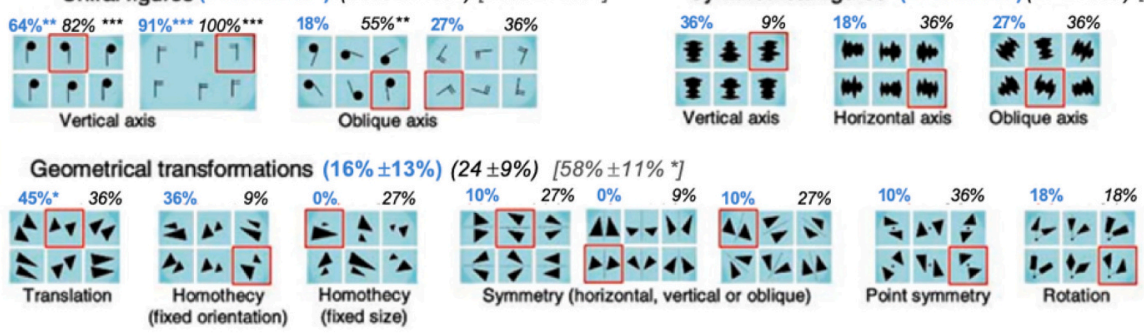

Significance at category level: ${ }^{*} p<0.00001$

Significance at trial level: ${ }^{\star \star \star} p<0.0001 ;{ }^{\star \star} p<0.005 ;{ }^{*} p<0.05$

Fig. 1. Methods. A. Example of a haptic trial. We created haptic sheets using the exact images of Dehaene et al.(Dehaene et al., 2006). Trials were engraved on a transparency sheet positioned in front of the participants. On each trial, participants were asked to pick the shape that they considered the most different from all the others, without any time constraints or feedback. B. Pictorial representation of all the trials of the deviant-figure detection task (Dehaene et al., 2006; V.Izard \& Spelke, 2009). Here, the trials are divided into seven geometric categories (e.g. Topology, Euclidean Geometry, etc.)(Dehaene et al., 2006; V.Izard \& Spelke, 2009), but trial order was randomized in the actual experiment. Each trial showed six ffgures that differed from each other in several features such as absolute orientation or size. Crucially, ffve of these ffgures deffned a speciffc geometric concept (indicated below each panel), whereas the sixth (surrounded in red) violated it. Average percent correct (with 95\% conffdence interval) is shown for each of the 7 geometric categories (blind participants: blue bold; blindfolded sighted participants: black italic; sighted in vision: gray italic). For the haptic performance of blind and sighted participants, average performance for each trial is also reported. Black asterisks depicts signiffcance levels against chance (16.6\%) using binomial test. (For interpretation of the references to color in this ffgure legend, the reader is referred to the web version of this article.) 
However, these results only address a limited range of geometric properties. Moreover, in this study the authors limited their investigation to the use of geometric cues during spatial navigation, while there is evidence suggesting that geometric knowledge may differently infiuence navigation and shape perception (Dillon et al., 2013; Spelke et al., 2010). To date, to the best of our knowledge, a systematic investigation of the role of visual experience on core geometric intuitions is still lacking: Does the emergence of core geometric intuitions stem from the visual experience of space, or do those intuitions refiect intrinsic properties of spatial cognition that are shared and consistent across sensory modalities?

To answer this question, we created a haptic version of the visual deviantffgure detection task previously used to test the perception of geometric properties in the visual modality by Dehaene and colleagues (Dehaene et al., 2006;Dillon et al., 2013;Izard \& Spelke, 2009) (seeFig. 1A-B). In each trial, $6 \mathrm{ffgures}$ were presented. All these ffgures differed in several aspects, such as for example in their size and/or orientation, but ffve of the ffgures shared a geometric property which the 6 th ffgure lacked (e.g. parallelism, symmetry; seeFig. 1B for all properties). Participants were instructed to choose the ffgure that they identiffed as the most different from all the others. Choosing the geometric deviant-ffgure would show that participants detected the relevant geometric property in spite of other types of spatial variations. Importantly, each geometrical concept was tested only once, precluding learning or strategy formation during the experiment itself.

We tested this haptic geometry task on a group of blind-from-birth participants and a group of sighted blindfolded controls. To test consistency within the responses of the blind participants, this group repeated the exact same haptic deviant-ffgure detection task in a second, separate experimental session. Sighted participants also participated in another experimental session in which they were tested again in the same exact task, albeit in vision (see Methods).

With this design, we can perform several comparisons. First, we can compare the performances of blind and sighted participants in touch. We can also compare performance when the participants from each group are using their dominant sensory modality for spatial exploration (touch and vision respectively). This design allows us to investigate whether geometric cues are used similarly across groups, and thus to what extent visual and haptic spatial shape perception/exploration share the same properties. So far, the available evidence with blind adults has yielded confiicting results. On one side, several studies documented that blind people, when directly asked, can learn and are able to correctly use various geometric concepts such as symmetry or mental rotations (Bauer et al., 2015;Carpenter \& Eisenberg, 1978; Cattaneo et al., 2014, 2010;Marmor \& Zaback, 1976;Occelli, Lacey, \& Sathian, 2016). These results show that blind adults can successfully acquire geometric foundations via touch, but whether they use the same geometric intuitions as sighted individuals to drive haptic spatial grouping and similarity judgments remain an open question. Some studies, for instance, highlighted differences between sighted and blind in spatial exploration strategies, with blind subjects preferentially activating an egocentric reference frame (self-to-object) and sighted subjects preferentially activating an allocentric one (objects-to-objects) (Chiesa, Schmidt, Tinti, \& Cornoldi, 2017; Iachini, Ruggiero, \& Ruotolo, 2014;Pasqualotto \& Proulx, 2012; Pasqualotto, Spiller, Jansari, \& Proulx, 2013;Röder, Rösler, \& Spence, 2004;Ruggiero, Ruotolo, \& Iachini, 2012;Schinazi, Thrash, \& Chebat, 2016). These differences might actually alter grouping strategies in the blind population, ultimately giving rise to different spatial judgments compared to the sighted population.

Secondly, this design allows us to compare performance in vision and touch directly in the sighted population, and ask whether responses converge across the two senses. To the best of our knowledge, geometric intuitions were never investigated in the tactile modality in sighted adults for small, 2-dimensional shapes. So far, studies on the properties of haptic space did not yet provide conclusive evidence regarding the comparison with visual space, probably also due to the fact that an in-depth investigation of the special conffguration classes (Feldman, 1997) driving haptic perception is still missing. Speciffcally, some results suggest that visual and haptic spaces do share the same properties with respect to spatial grouping (Haggard \& Giovagnoli, 2011;Overvliet, Krampe, \& Wagemans, 2012, 2013;Serino, Giovagnoli, de Vignemont, \&
Haggard, 2008; Verlaers, Wagemans, \& Overvliet, 2015), while other results document differences in interpreting spatial relations among objects between the two sensory modalities (Cuijpers, Kappers, \& Koenderink, 2003; Kappers, 1999, 2003, 2004;Kappers \& Koenderink, 1999).

Through our deviant-ffgure detection task, we presented participants with a wide range of geometric concepts (Fig. 1B), thus allowing to extract performance proffles and to evaluate the convergence and divergence of responses across vision and touch. Observing similar patterns of performance (i.e., high correlations between haptic and visual performances) irrespective of sensory experience (blind vs. sighted) and of the sensory modality used (vision or touch) would suggest the existence of an amodal core-system of geometry that applies to small, 2-dimensional ffgures. Alternatively, observing different patterns of performance between groups and/or sensory-inputs would suggest the presence of two distinct systems of modality-speciffc geometric representations.

Finally, we used two different measures to examine the role of formal geometric education on the performance of blind participants. First, at the end of the whole experiment, we collected self-reports on the years blind participants were exposed to formal geometry education (see Methods). Second, we compared the performance of our blind participants in touch with that of uneducated Mundurucu adults (Dehaene et al., 2006) and pre-school children performing the same task in vision (Izard \& Spelke, 2009).

\section{Methods}

\subsection{Participants}

We tested eleven blind adults (four females; mean age $41.18 \pm 9.8$ ) in the haptic deviant-figure detection task. None of them had other known neurological diseases. Among them, nine were congenitally blind and two had minimal light perception only until very early in life, but have no visual memories nor form perception relevant to the task (see Table S1 for blind characteristics). The only exclusion criterion for our blind participants was that they should not present other sensory, physical or cognitive deficits. No participant was excluded based on these criteria. Eleven age- and gender-matched sighted adults were also tested (mean age $40.45 \pm 12.15$ ) and were blindfolded throughout the tactile experiment. The Hebrew University's ethics committee for research involving human subjects approved the experiment, and written informed-consent was obtained from each participant. Participants were reimbursed for their participation.

\subsection{Apparatus and stimuli}

Experimental stimuli were identical to those used in previous visual studies using the same exact task (Dehaene et al., 2006; Izard \& Spelke, 2009). However, we created a tactile version of all the stimuli in order to allow their haptic exploration. Stimuli were drawn on transparency sheets using a specially designed rubberized writing board (Sewell raised line drawing kit). In this way, lines and shapes were engraved on the transparency sheet and could be explored haptically by participants (Fig. 1A, Supplementary Movie S1). In each trial (i.e., one transparency sheet), six different ffgures were presented. In order to make clear borders between the six ffgures, each one of them was embedded into a frame of a different texture (Fig. 1A, Supplementary Movie S1). All ffgures forming a trial differed between each other in terms of several spatial features such as absolute orientation or size. Five of these ffgures shared a speciffc geometric property (e.g. quadrilaterals), while the sixth ffgure deviated from it (e.g., triangle). The experiment consisted of 43 experimental trials and 4 training trials (seeFig. $1 \mathrm{~B}$ for all the trials). The 4 training trials aimed at making sure that participants understood the task and were able to perceive all the shapes in the haptic sheets (i.e., the raised lines were above the haptic acuity threshold). These trials were built in the same way as the experimental trials but did not test a geometric property (texture, orientation, numerosity and orientation). The 43 experimental trials were divided in seven higher-order categories deffned in a previous study using the same paradigm in the visual modality (Dehaene et al., 2006): Topology (e.g., connectedness, continuity, 
closure), Euclidean Geometry (e.g., straight lines, alignment, parallelism), Geometrical Figures (e.g., quadrilaterals, triangles, circle), Metric Properties (distance and proportions), Chiral Figures (mirror images, rotated or not), Symmetry Figures (complex ffgures in which the symmetry of shapes was manipulated in the vertical, horizontal or oblique axes) and Geometrical Transformations (spatial relations between two geometrical ffgures, such as translations or rotations).

\subsection{Procedure}

Each participant was tested individually by the experimenter and was instructed to freely explore all of the six ffgures presented in each trial, and indicate the one he/she thought was the most different from all the others, without any time constraints. Sighted participants were blindfolded throughout the haptic experiment. Trial presentation was pseudo-randomized across participants, such that trials testing similar concepts were not presented one after the other. Before starting the experiment, all participants were presented with 4 training trials in which they received feedback on their responses. During the experimental task, no response feedback was provided. Breaks were given approximately every 10-15 trials for blind participants, every 4-9 trials for blindfolded sighted participants, or upon participant's request. Blind participants completed the experimental session in $\sim 60$ minwhile sighted blindfolded participants needed $\sim 120 \mathrm{~min}$ to complete the task. For this reason, for sighted participants the full experimental session was divided into two parts that were completed within a time-interval of maximum $24 \mathrm{~h}$, whereas blind participants completed the whole experiment in one session only.

In order to test the consistency of performance, a second experimental session (i.e., repeating the whole experimental procedure) was carried out only by blind participants, within a time-interval of 1-3 weeks (mean: 13.45 days, $\mathrm{SE}=2.42$ ) from the first session, depending on the participants' availability. Finally, sighted participants came for an additional experimental session that was carried out after the previous session (3-84 days). During this session participants were not blindfolded and were asked to perform the exact same task, using the exact same stimuli engraved on transparency sheets which were now presented to them visually rather than haptically. For technical reasons, we could not analyze data from one participant, therefore results on vision are reported for 10 participants.

\subsection{Assessing formal geometry education in blind participants}

To exclude any effect of formal geometric education on the results of blind participants, at the end of the second session, we asked participants to selfdeclare how many years of formal geometry education they had received. Speciffcally, we asked our blind participants to report until which age they studied geometry in school. All participants were exempted at some point from studying geometry before the end of high-school, and could only provide a rough estimation on when exactly they were exempted. Thus, responses of participants were divided into 3 categories and we assigned a categorical value to each one of them: 0 if participants declared they had never studied geometry $(n=1) ; 1$ if participants declared they had studied geometry only at elementary school $(n=4)$; and 2 if participants declared they had studied geometry also in high-school until they were exempted $(n=6)$.

\section{Results}

\subsection{Haptic deviant-ffgure detection task}

Results from the haptic deviant-ffgure detection task show that blind-frombirth participants identiffed the geometric deviant in most trials and categories. Their overall performance was signiffcantly above the chance level of $16.6 \%$ (binomial test against chance: $p$-value $<0.0001$ ) and was comparable to the overall performance of sighted but blindfolded participants (blind: average $48 \% \pm 7 \%$ [ $95 \%$ conffdence interval]; Sighted blindfolded: average $52 \% \pm 6 \%$; Fig. 2A). As shown in Fig. 1B, both blind-from-birth and sighted blindfolded participants selected the geometric deviant shape well above the chance level of $16.6 \%$ in the categories of topology (e.g., closure or connectedness), Euclidean geometry (e.g., curvature, alignment of points to lines, parallelism) and geometrical ffgures (e.g., quadrilateral, triangle, or circle). Both groups experienced more difffculties, but still achieved overall higher than chance performance, in two other categories. With metric properties, participants mostly successfully detected distance relations among separate elements of a given ffgure, namely trials testing absolute distances (e.g. equidistance trial), but failed in trials involving metric relations within the same shape, namely relative distance trials (e.g., middle of segment; ffxed proportions trials). In the chiral ffgures category (i.e., distinguishing a shape from its mirror image), participants also performed above chance overall, but while both groups easily identiffed the mirror image as the deviant in trials in which all the ffgures were presented with ffxed orientation (vertical axis trials), both had more difffculties and mostly failed to spontaneously use chiral cues for the identiffcation of the deviant in trials in which the orientation differed between the stimuli (oblique axes trials), thus requiring more complex mental rotations.

This remarkable performance did not extend to the categories testing symmetrical ffgures (symmetry of ffgures in the horizontal, vertical or oblique axis) and geometrical transformations (trials requiring the detection of spatial relations between two geometrical ffgures, such as translation and rotation), where both groups did not overcome chance level (seeFig. 1B for means and single trials results).

We then directly compared the performance of blind-from-birth and sighted blindfolded subjects when both used touch. Despite blind participants' more extensive experience in haptic exploration, which was reflected by their much quicker overall response time to complete the task compared to the sighted blindfolded participants (average overall response time: Blind $=63.47 \mathrm{~min}$; Sighted blindfolded $=118.86 \mathrm{~min}$; -value $<0.001)$, a Wilcoxon test showed no difference in overall accuracy between groups ( $\mathrm{W}=$ 74.5, p-value $<0.4$;Fig. 2A). This result was further corroborated by a series of Kruskal-Wallis tests showing that the performance of the two populations did not signiffcantly differ in any of the geometric categories (Fig. 2A; allpvalues $>0.06$ ). In addition, and importantly, the performance of blind and sighted blindfolded participants was highly correlated across trials (Spearman correlation, rho $=0.76$; p-value $<0.0001$;Fig. $2 \mathrm{~B}$ ). Speciffcally,Fig. $2 \mathrm{~B}$ shows the average geometric selection rate for each of the experimental trials in blind ( $x$-axis) and sighted (blindfolded) participants ( $y$-axis). The high correlation of performance between groups means that both groups tended to succeed/fail on the same trials.

\subsection{Consistency in blind participants' performance}

To further validate our results in the blind population, we repeated the whole experimental procedure on this group of participants in a separate experimental session (mean interval between sessions: 13.45 days, $\mathrm{SE}=2.42$ ). A Spearman correlation test on the average performance in each experimental trial in the two sessions revealed a highly consistent performance of the blind group (rho $=0.87$, $p$-value $<0.0001$; see Supplementary Fig. 1; see Supplementary Analyses 1 for full analyses). Additionally, a Wilcoxon test conffrmed no statistical difference between the overall performance of blind participants in the second experimental session and the overall performance of sighted participants in the haptic deviant-ffgure detection task $(\mathrm{W}=67 ; \mathrm{p}$-value $=0.7)$.

\subsection{Performance in vision vs. touch}

In a separate experimental session, sighted participants performed the exact same task in the visual modality alone, using exactly the same stimuli (due to technical reasons, we could not analyze results from one participant). This session was always held after the tactile session (in which they did not receive any feedback on their answers). This was done to minimize the use of visual imagery during tactile exploration (range between the tactile and the visual experimental sessions: 3-84 days depending on the availability of participants). Results revealed that the overall visual performance of sighted participants was entirely comparable to previous data with 


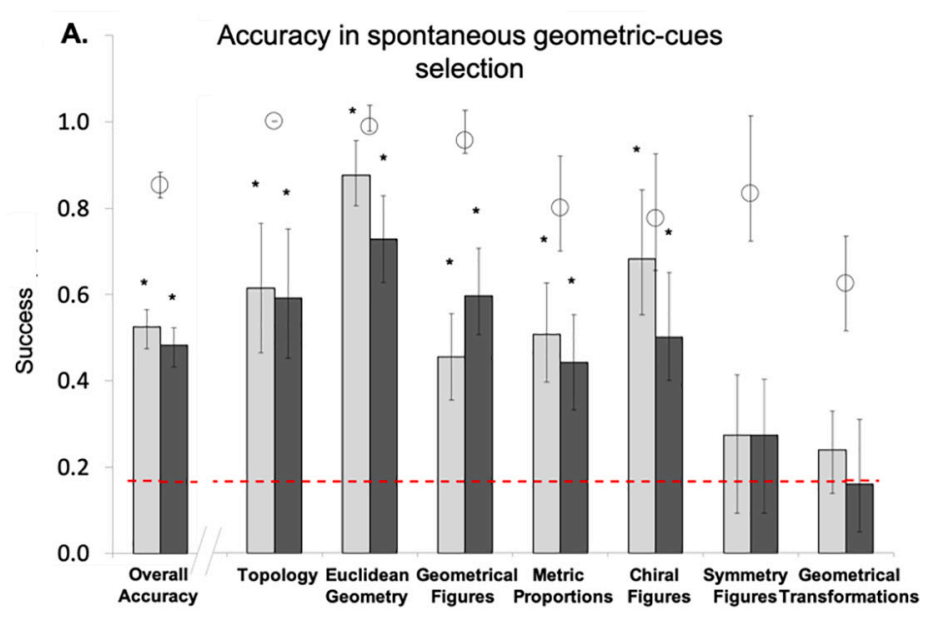

\section{B. Correlation between haptic performances of sighted and blind-from-birth adults}

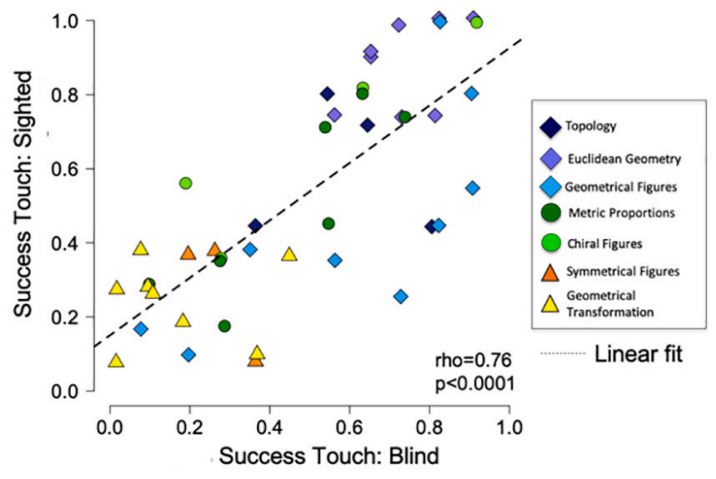

Touch: Sighted (blindfolded) $n=11 \square$ Touch: Blind-from-birth $n=11 \bigcirc$ Vision: Sighted $n=10$

Chance Level (16.6\%) * $p<0.0001$ (vs. Chance Level)

\section{Correlation between performance in vision and in touch (blind)}

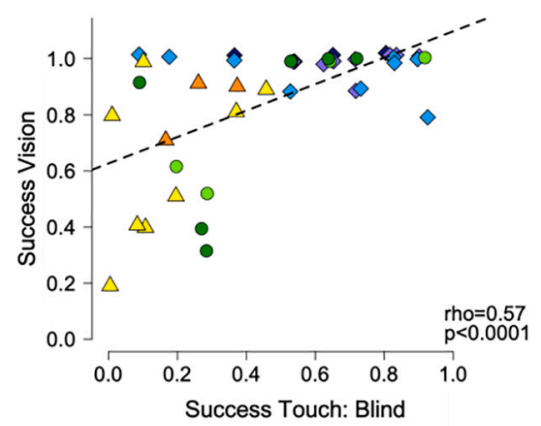

E. Correlation between performances of sighted Amazonian adults and blindfrom-birth adults

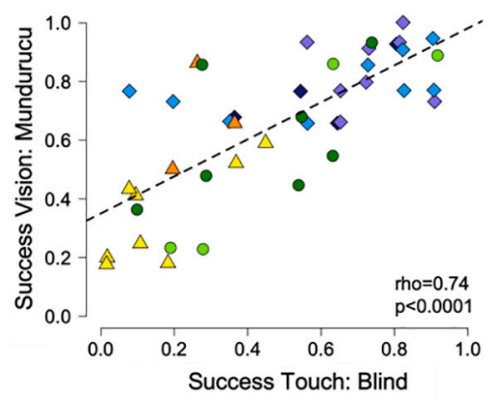

D. Correlation between performance in vision and in touch (sighted blindfolded)

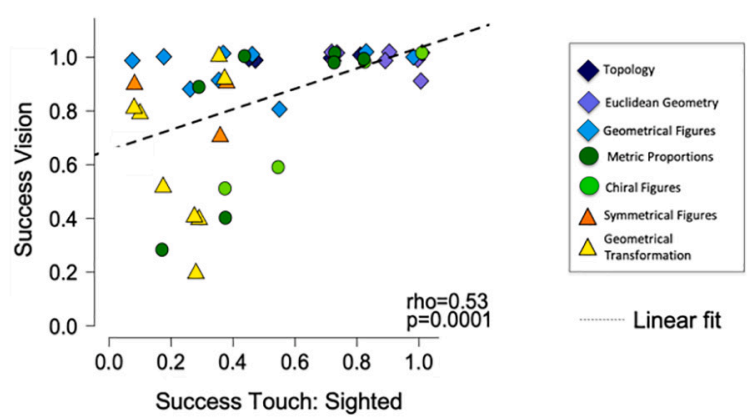

F. Correlation between performances of sighted pre-school children and blindfrom-birth adults

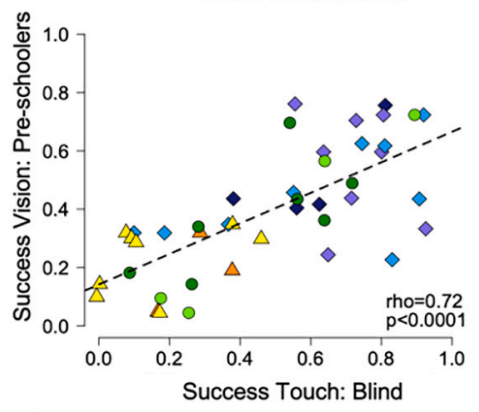

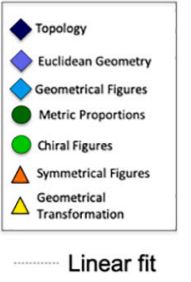

Linear fit

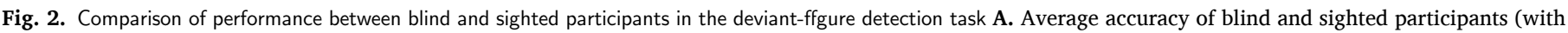

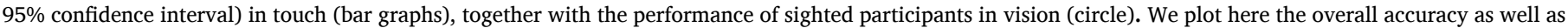

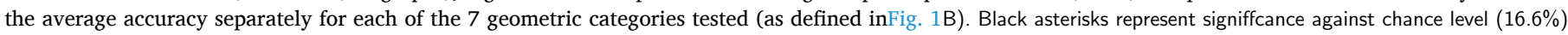

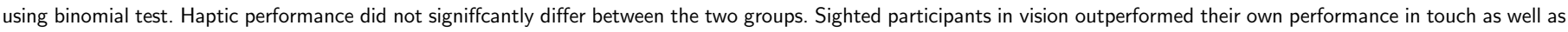

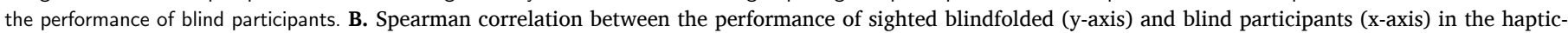

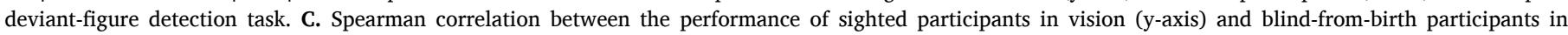

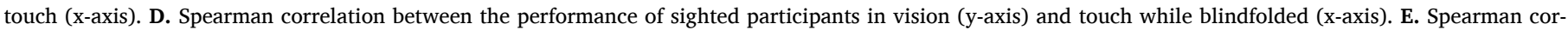

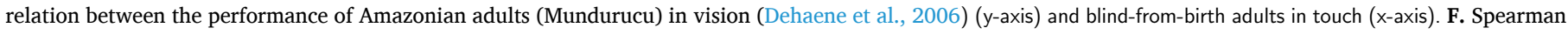

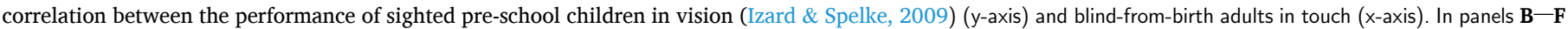

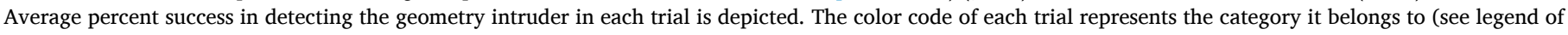
the figures and seeFig. 1B for details). 
sighted adults in the same task (Dehaene et al., 2006), but it was significantly better than their own performance in touch $(\mathrm{W}=110, p$-value $=$ $0.0001)$ as well as that of blind-from-birth participants (W $=110$, pvalue $=0.0001$ ) (Fig. 2A). This visual geometric advantage was also observed within the different geometric categories (Fig. 2A and see Supplementary Analysis 2 and Supplementary Fig. 2 for full analyses results). Crucially, however, across trials visual performance was again highly positively correlated with the performances of sighted but blindfolded participants (rho $=0.53$, p-value $<0.0001$ ) as well as blind-from-birth participants in the haptic task (rho $=0.57$, p-value $<0.0001$ ) (Fig. $2 \mathrm{C}$ D). In other words, the same trials tended to be easier or harder in both visual or haptic deviant-ffgure detection tasks.

\subsection{Examining the role of formal geometric education in blind participants' performance}

To examine the potential role of formal geometric education in the performance of blind participants in the haptic deviant-ffgure detection task, at the end of the second experimental session, we asked participants to self-report the years of formal geometric education that they received in school until exemption (none of the participants completed the high-school geometry curriculum; see Methods for details). No correlation was found between overall accuracy in our deviant-ffgure detection test and self-reported years of formal geometry education ( $r$ ho $=0.21$; $\mathrm{p}$-value $=0.5$ ).

As an additional test for the role of formal education, we compared the results of our blind participants in touch with previous data documenting the performance in the same task in the visual modality in two groups: (1) sighted adults coming from an Amazonian group, the Mundurucu, who lacked any formal geometric education and also lack lexical terms for the geometric concepts tested in our task (Dehaene et al., 2006) and (2) U.S. preschoolers (Izard \& Spelke, 2009). Across trials, we found signiffcant Spearman correlations between the performance of blind adults in touch and both Amazonian adults (rho $=0.74$, p-value $<0.0001$ ) and preschoolers in vision (rho $=$ 0.72 , p-value $<0.0001$ ) (Fig. $2 \mathrm{E}-\mathrm{F}$ ). These latter results further highlight the consistent nature of geometric intuitions across sensory modalities. The overall performance of pre-school children in vision and of blind-from-birth adults in touch did not differ (Kruskal-Wallis chi-squared $=1.79$, $\mathrm{df}=1$, $\mathrm{p}$-value $=$ 0.2). Mundurucu adults however outperformed blind participants (Kruskal-Wallis chi-squared $=11.65, \mathrm{df}=1, \mathrm{p}$-value $=0.0006$ ).

Taken together, these results further exclude a crucial role of formal education in the emergence of spontaneous geometric intuitions. Moreover, they suggest that geometric intuitions are strengthened by life-long spatial exploration carried out through vision rather than touch.

\section{Discussion}

In the present study, we tested whether visual experience is necessary for analyzing ffgures according to their geometrical properties. Three main conclusions emerged from our ffndings: 1) In the absence of experience with vision, blind-from-birth participants can analyze haptic ffgures according to a wide range of geometric properties. 2) The performance of sighted and blind-from birth participants was highly correlated across items, independently of the sensory input modality. This indicates that the geometric concepts that were harder to detect for the blind (such as symmetry and complex geometric transformations) were also harder to detect for sighted participants, both in touch and vision. This in turn suggests that the two sensory modalities evoke similar geometric intuitions, ultimately providing supporting evidence in favor of the existence of an abstract, amodal system of geometrical concepts. 3) Geometric selection was overall more frequent in the visual than in the haptic modality, suggesting that sensory-speciffc perceptual/spatial properties might play a crucial role in ffne tuning the saliency of geometric cues during visual versus haptic processing. In the following paragraphs, we further discuss the implications of these three conclusions.

\subsection{An amodal system of core geometry intuitions}

The ffrst and most remarkable result of the present study is that blind-frombirth adults consistently use many geometric concepts (e.g. parallelism, quadrilaterals, angles, topology, seeFig. 1B for all trials) to identify the haptic deviant. These results in turn demonstrate for the ffrst time the existence of a set of geometric intuitions that emerge independently of visual experience and that drive spatial grouping and spatial reasoning even when touch, rather than vision, has been the dominant sensory modality for spatial exploration across the life span.

Whereas blind-from-birth participants spontaneously detected the geometric deviant in most trials, they failed to do so in trials requiring geometric transformations of symmetry or rotation (whether internal to a shape, or as a relation between two shapes;Fig. 1B). Interestingly, all participants, whether sighted or blind, and whether performing the task in vision or touch, performed worst on these speciffc trials, both in our data set (Fig. 1B;Fig. 2; Supplementary Fig. 2) as well as in previously published experiments using the same paradigm (Dehaene et al., 2006; V.Izard \& Spelke, 2009;Izard et al., 2011). More generally, participants showed a similar proffle of performance across the different individual trials, which was unraveled by strong signiffcant correlations across populations and sensory modalities (Fig. 2C-F). One possibility is that such correlations arise from shared general-domain processes unrelated to geometry (i.e., spatial reasoning or working memory). However, two aspects of our task argue against this interpretation. First, while all experimental trials differ in their geometric or spatial content, they did not differ in terms of the (spatial) reasoning involved. Second, participants did not systematically fail to select the geometric deviant in the most perceptually complex trials (seeFig. 1B for a visual representation of all experimental trials), thus excluding the hypothesis that differences in working memory requirements among trials might underlie our results. The strong correlations reported here thus appear to provide encouraging evidence towards the existence of an amodal system of geometric intuitions that can be accessed through both the visual and haptic modalities.

This latter proposal ffts with previous ffndings in visual-haptic object recognition experiments documenting shared brain mechanisms subtending both visual and haptic geometrical shape-information processing (Amedi, Raz, Azulay, Malach, \& Zohary, 2010; James et al., 2002; Lacey, Tal, Amedi, \& Sathian, 2009). Speciffcally, it was proposed that the LOtv, a region in the lateral occipital cortex, is involved in all tasks of object shape processing, independently of the sensory modality used (vision, sound, and $3 \mathrm{D}$ or $2 \mathrm{D}$ touch), in both sighted (Amedi et al., 2010;Amir, Biederman, \& Hayworth, 2011;James, Kim, \& Fisher, 2007;Kubilius, Wagemans, \& Op de Beeck, 2014;Lacey et al., 2009;Mancini, Bolognini, Bricolo, \& Vallar, 2011; Snow, Strother, \& Humphreys, 2014) and blind populations (Amedi et al., 2010;Dormal et al., 2018;Lacey et al., 2009;Peelen, He, Han, Caramazza, \& Bi, 2014;Ptito et al., 2012). Overall then, these previous studies might start to provide a shared neural substrate for the spontaneous detection of geometric concepts. However, its speciffc characteristics as well as those of the related processing network still need to be further investigated using neuroimaging techniques. Future studies should for instance disentangle whether the neuronal populations underlying judgments about the geometric properties of shapes are multisensory (visuo-tactile) or sensory-speciffc (visual; tactile). The former outcome would conffrm the truly amodal nature of the geometry system (see for instance (Tal \& Amedi, 2009) for initial results in this direction). The latter outcome would instead suggest two distinct modality-speciffc geometric networks, albeit largely overlapping and interconnected. Note that so far, only a small set of studies have started to investigate mathematical processing in blind subjects, and shown that they tend to activate math-responsive circuits largely similar to those of sighted subjects, often with the additional recruitment of occipital cortex (Amalric \& Dehaene, 2016;Amalric, Denghien, \& Dehaene, 2018;Kanjlia, Lane, Feigenson, \& Bedny, 2016;Togoli, Crollen, Arrighi, \& Collignon, 2020).

Importantly, and in line with previous evidence in the sighted population (Dehaene et al., 2006; Izard \& Spelke, 2009), the present ffndings also suggest that formal geometric education does not carry a crucial role, if any, in driving the detection of simple geometric cues in blind participants. We 
reported a lack of correlation between the overall performance of blind participants in the haptic deviant-ffgure detection task and their self-declared years of formal geometry education (none of the blind participants ffnished the school geometry curriculum, see details in methods). Furthermore, we showed that the performance in the same task carried out in vision by both preschool children and sighted Amazonian adults lacking any geometric education was highly correlated with the blind performance in touch (Fig. 2E-F).

\subsection{Differences between visual and tactile geometrical exploration}

The present data revealed that sighted participants were more sensitive to geometric properties when tested with vision than with touch. In touch, their performance was entirely comparable to that of blind adults, but in vision they selected the geometric deviant with better accuracy (Fig. 2A), albeit still showing relative difffculties in the same groups of trials (Fig. 2C-D). It is important to highlight that this difference cannot be attributed to differences in perceptibility between haptic and visual stimuli. We indeed took various precautions to ensure that the haptic stimuli were perfectly perceptible by touch: First, we performed various qualitative pilots prior to the start of the experiment to ensure that participants could perceive all the shapes within the haptic sheets, namely that the raised lines were above the tactile acuity threshold. Second, we checked that stimuli were above acuity threshold for all our participants in two ways: by asking them to perform 4 training trials at the beginning of the experiment (see Methods for details) and by monitoring participants' exploration on-line, during the experiment (all participants in both groups fully explored the haptic sheets).

Thus, although more work will be needed to fully conffrm this point, the gap in geometry selection between the two modalities seems to suggest that the system for spontaneous geometric selection during spatial exploration is more efffcient in vision than touch. Note, however, that the reasons behind the difffculties of our sighted participants in the haptic compared to the visual version of the task might be also related to their reduced experience in haptic exploration - refiected in the much longer time needed by sighted participants to complete the haptic task compared to blind-from-birth participants. ${ }^{2} \mathrm{An}$ additionally, not mutually exclusively possibility for this longer experimental time in sighted blindfolded participants, might be related to the fact that they possibly tried to visually imagine the haptic ffgures, which might have perhaps facilitated their answers but slowed them in time. Overall, we cannot exclude that after speciffc and prolonged haptic training, sighted participants would show a performance in the haptic deviant-ffgure detection task more similar to their visual one, perhaps however, strongly mediated by visual imagery.

Nonetheless, spontaneous geometric selection was also better when sighted participants used vision than when blind adults used touch, even though blind adults have relied mainly on touch to explore their spatial environment across their whole lifespan. This in turn strengthens the conclusion that geometric selection is facilitated in the visual modality, ultimately strengthening previous results reporting different behavioral (and transfer) efffciency between sensory modalities in certain tasks (McGovern, Astle, Clavin, \& Newell, 2016).

One possible interpretation might be that various geometric concepts simply do not develop as a consequence of haptic rather than visual spatial experiences. However, several previous studies showed that blind participants do possess and can successfully master the geometric concepts which they failed to spontaneously detect in the current work. For instance, blind

\footnotetext{
${ }^{2}$ To test potential training facilitation in geometric selection in sighted blindfolded participants, we performed an additional analysis comparing their performance in the two separate sessions which were held in two consecutive days (see Methods). Our aim was to check whether we observed a tendency for increased geometric selection in the second part of the experiment (i.e., after participants acquired some experience with tactile exploration). Note that the first experimental session in sighted, lasted more or less the same amount of time needed by blind to end the whole task. These analyses revealed no difference in geometric performance between the two parts of the experiment ( $p=$ 0.9 ), despite a tendency for being faster in the second compared to the first part of the experiment.
}

participants succeeded in perceiving symmetry relations when directly comparing symmetrical and non-symmetrical ffgures (Bauer et al., 2015; Cattaneo et al., 2014, 2010) and in performing mental rotations under more focused experimental conditions (Carpenter \& Eisenberg, 1978;Marmor \& Zaback, 1976;Occelli et al., 2016). Some of these studies even showed that the neural representations of certain features such as symmetry are shared between the blind and sighted populations (Bauer et al., 2015). This evidence, in turn, discourages the conclusion that geometric concepts simply cannot be acquired through touch.

Another option is to interpret the difference in accurate geometric selection between vision and touch as evidence in favor of the existence of two separate sensory-speciffc systems of geometric intuitions, which nonetheless are characterized by partially shared spatial exploration properties that underlie similar (to some extent) geometric selection outcomes. However, the strong correlations between visual and haptic performance discussed in the section above (Fig. 2C-D), together with known differences between haptic-driven and visually-driven sensorimotor spatial explorations, ultimately leading to different explorative behaviors between vision and touch (Chiesa et al., 2017;Pasqualotto \& Newell, 2007;Postma, Zuidhoek, Noordzij, \& Kappers, 2007, 2008) seem to suggest a slightly different conclusion. Speciffcally, this latter evidence may not discourage the existence of an amodal, visuo-tactile shared system of geometry, but rather suggests the presence of certain sensoryspeciffc constraints. Such constraints differentially reffne the shared system properties, facilitating for instance visual over haptic geometric selection. In line with this conclusion, we found that uneducated Amazonian adults tested with vision outperformed blind adults in touch, ultimately suggesting that a lifetime of visual sensorimotor spatial exploration may enhance geometric intuitions. More in detail, the sequential nature of tactile exploration as opposed to the parallel nature of visual exploration is known to facilitate local over holistic spatial representations, as well as the preferential activation of an egocentric (self-to-object) rather than an allocentric (object-to-object) reference frame, with this latter preference particularly evident in blind participants during haptic tasks (Chiesa et al., 2017; Iachini et al., 2014;Pasqualotto et al., 2013;Pasqualotto \& Proulx, 2012;Röder et al., 2004;Ruggiero et al., 2012;Schinazi et al., 2016). And indeed, the geometric properties that were the hardest to detect by blind participants were exactly those requiring the adoption of holistic spatial representations and an allocentric reference frame, i. e. trials requiring holistic shapes comparisons (e.g., symmetry transformations), or a direct comparison either between shapes (e.g., geometrical transformation trials), parts of the same shape (e.g. relative distance or proportion) or a shape and its context (e.g., mental rotation). In light of these considerations and based on the current result that many spatial geometric intuitions are indeed shared between vision and touch, future studies may investigate the extent to which training speciffcally tailored to spatial exploration strategies may render these geometric concepts more spontaneously accessible to the blind, ultimately thinning or even eliminating the differences with the visually-driven geometric selection.

\subsection{Conclusions}

The present study shows that a wide range of spatial geometric intuitions can develop independently of visual experience and drive judgments about shapes in a largely similar manner in blind and sighted adults, independently of the main sensory-modality used for spatial exploration (touch or vision). This ffnding provide encouraging evidence to the hypothesis that geometrical concepts may belong to a "core knowledge" system which is either innate or arises largely invariantly from both formal education and sensoryspecific spatial experience. However, even though visual and haptic performances were highly correlated, we observed that vision was more efficient than touch for detecting geometric deviants. This in turn highlights that shared cognitive and perceptual systems may not develop exactly the same behavioral properties across sensory systems due to sensory-specific constraints, and thus, in turn, some sensory-modalities may be intrinsically more efficient to process a specific type of stimuli/computation. We suggest that in case of the core-system of geometry, such differences might stem from intrinsic differences between 
haptically-driven and visually-driven explorative/perceptual behaviors, rather than from differences in the mastering or possession of geometric concepts per se. Taken together, these results contribute new evidence to the recurrent debate on the origins of geometry: not only do humans rely on abstract geometric cues in their judgments about shapes even in the absence of formal education (Dehaene et al., 2006; Dillon et al., 2013; Izard et al., 2011;Izard \& Spelke, 2009;Lourenco \& Huttenlocher, 2008), but they largely do so also in the absence of visual experience. Our results may also have important educational implications: in agreement with the initial statement by the outstanding blind mathematician Emmanuel Giroux, they suggest that people who are blind, regardless of their visual impairment, share a solid universal platform of mathematical intuitions and may thrive in mathematics.

Supplementary data to this article can be found online at https://doi. org/10.1016/j.cognition.2021.104716.

Authors contributions

$\mathrm{BH}, \mathrm{TB}, \mathrm{SD}$ and $\mathrm{AA}$ conceptualized the study. $\mathrm{BH}, \mathrm{TB}$ and $\mathrm{AA}$ designed the study. $\mathrm{BH}$ and $\mathrm{TB}$ gathered the data. $\mathrm{BH}$ performed statistical analyses. $\mathrm{SD}$ and $\mathrm{VI}$ shared, to be used in the study, Mundurucu and US pre-schoolers data, respectively. $\mathrm{BH}, \mathrm{TB}, \mathrm{AA}, \mathrm{SD}, \mathrm{VI}$ wrote the manuscript.

\section{Acknowledgements}

This work was supported by aEuropean Research Councilgrant (grant number773121) to AA; a Joy Venture grant to AA.

\section{References}

Amalric, M., \& Dehaene, S. (2016). Origins of the brain networks for advanced mathematics in expert mathematicians. In Proceedings of the National Academy of Sciences (p. 201603205). https://doi.org/10.1073/pnas.1603205113.

Amalric, M., Denghien, I., \& Dehaene, S. (2018). On the role of visual experience in mathematical development: Evidence from blind mathematicians. Developmental Cognitive Neuroscience, 30, 314-323. https://doi.org/10.1016/j.dcn.2017.09.007.

Amedi, A., Raz, N., Azulay, H., Malach, R., \& Zohary, E. (2010). Cortical activity during tactile exploration of objects in blind and sighted humans. Restorative Neurology and Neuroscience, 28(2), 143-156. https://doi.org/10.3233/RNN-2010-0503.

Amir, O., Biederman, I., \& Hayworth, K. J. (2011). The neural basis for shape preferences. Vision Research, 51(20), 2198-2206. https://doi.org/10.1016/j.visres.2011.08.015.

Amir, O., Biederman, I., \& Hayworth, K. J. (2012). Sensitivity to nonaccidental properties across various shape dimensions. Vision Research, 62, 35-43. https://doi.org/10.1016/j. visres.2012.03.020.

Amir, O., Biederman, I., Herald, S. B., Shah, M. P., \& Mintz, T. H. (2014). Greater sensitivity to nonaccidental than metric shape properties in preschool children. Vision Research, 97, 83-88. https://doi.org/10.1016/j.visres.2014.02.006.

Bauer, C., Yazzolino, L., Hirsch, G., Cattaneo, Z., Vecchi, T., \& Merabet, L. B. (2015) Neural correlates associated with superior tactile symmetry perception in the early blind. Cortex, 63, 104-117. https://doi.org/10.1016/j.cortex.2014.08.003.

Biederman, I., Yue, X., \& Davidoff, J. (2009). Representation of shape in individuals from a culture with minimal exposure to regular, simple artifacts: Sensitivity to nonaccidental versus metric properties. Psychological Science, 20, 1437-1442. https://doi.org/ 10.1111/j.1467-9280.2009.02465.x.

Carpenter, P. A., \& Eisenberg, P. (1978). Mental rotation and the frame of reference in blind and sighted individuals. 23(2), 117-124.

Cattaneo, Z., Bona, S., Bauer, C., Silvanto, J., Herbert, A., Vecchi, T., \& Merabet, L. (2014). Symmetry detection in visual impairment: Behavioral evidence and neural correlates. Symmetry, 6(2), 427-443. https://doi.org/10.3390/sym6020427.

Cattaneo, Z., Fantino, M., Silvanto, J., Tinti, C., Pascual-Leone, A., \& Vecchi, T. (2010). Symmetry perception in the blind. Acta Psychologica, 134(3), 398-402. https://doi.org 10.1016/j.actpsy.2010.04.002.

Cheng, K., \& Newcombe, N. S. (2005). Is there a geometric module for spatial orientation? Squaring theory and evidence. Psychonomic Bulletin and Review, 12(1), 1-23. https:// doi.org/10.3758/BF03196346.

Chiesa, S., Schmidt, S., Tinti, C., \& Cornoldi, C. (2017). Allocentric and contra-aligned spatial representations of a town environment in blind people. Acta Psychologica, 180, 8-15. https://doi.org/10.1016/j.actpsy.2017.08.001.

Cuijpers, R. H., Kappers, A. M. L., \& Koenderink, J. J. (2003). The metrics of visual and haptic space based on parallelity judgements. Journal of Mathematical Psychology, 47(3), 278-291. https://doi.org/10.1016/S0022-2496(03)00004-X.

Dehaene, S., Izard, V., Pica, P., \& Spelke, E. (2006). Core knowledge of geometry in an Amazonian indigene group. Science, 311(5579), 381-384. https://doi.org/10.1126/ science.1121739.

Descartes, R. (1886). La géométrie. Hermann.

Diderot, D. (1749). Lettre sur les aveugles, a l'usage de ceux qui voyent.
Dillon, M. R., Huang, Y., \& Spelke, E. S. (2013). Core foundations of abstract geometry. Proceedings of the National Academy of Sciences of the United States of America, 110 (35), 14191-14195. https://doi.org/10.1073/pnas.1312640110.

Dormal, G., Pelland, M., Rezk, M., Yakobov, E., Lepore, F., \& Collignon, O. (2018). Functional preference for object sounds and voices in the brain of early blind and sighted individuals. Journal of Cognitive Neuroscience, 30(1), 86-106. https://doi.org/ 10.1162/jocn_a_01186.

Feldman, J. (1997). Regularity-based perceptual grouping. Computational Intelligence, 13, 582-623. https://doi.org/10.1111/0824-7935.00052.

Feldman, J. (2007). Formation of visual "objects" in the early computation of spatial relations. Perception \& Psychophysics, 69(5), 816-827. https://doi.org/10.3758/ BF03193781.

Gallistel, C. R. (1990). The organization of learning. Cambridge, MA, US: MIT Press.

Gibson, B. M., Lazareva, O. F., Gosselin, F., Schyns, P. G., \& Wasserman, E. A. (2007) Nonaccidental properties underlie shape recognition in mammalian and nonmammalian vision. Current Biology, 17, 336-340. https://doi.org/10.1016/j.cub.2006.12.025.

Haggard, P., \& Giovagnoli, G. (2011). Spatial patterns in tactile perception: Is there a tactile ffeld? Acta Psychologica, 137(1), 65-75. https://doi.org/10.1016/j. actpsy.2011.03.001.

Iachini, T., Ruggiero, G., \& Ruotolo, F. (2014). Does blindness affect egocentric and allocentric frames of reference in small and large scale spaces? Behavioural Brain Research, 273, 73-81. https://doi.org/10.1016/j.bbr.2014.07.032.

Izard, V., \& Spelke, E. S. (2009). Development of sensitivity to geometry in visual forms. Human Evolution, 24(3), 213-248.

Izard, V., Pica, P., Dehaene, S., Hinchey, D., \& Spelke, E. (2011). Geometry as a Universal Mental Construction. Space, Time and Number in the Brain (Vol. 1). Elsevier Inc.. https://doi.org/10.1016/B978-0-12-385948-8.00019-0

James, T. W., Humphrey, G. K., Gati, J. S., Servos, P., Menon, R. S., \& Goodale, M. A. (2002). Haptic study of three-dimensional objects activates extrastriate visual areas. Neuropsychologia, 40(10), 1706-1714. https://doi.org/10.1016/S0028-3932(02) 00017-9.

James, T. W., Kim, S., \& Fisher, J. S. (2007). The neural basis of haptic object processing. Canadian Journal of Experimental Psychology/Revue Canadienne de Psychologie Expérimentale, 61(3), 219-229. https://doi.org/10.1037/cjep2007023.

Kanjlia, S., Lane, C., Feigenson, L., \& Bedny, M. (2016). Absence of visual experience modiffes the neural basis of numerical thinking. Proceedings of the National Academy of Sciences, 113(40), 11172-11177. https://doi.org/10.1073/pnas.1524982113.

Kant, I. (1998). In P. Guyer, \& A. W. Wood (Eds.), Critique of Pure Reason. Cambridge University Press.

Kappers, A. M. L. (1999). Large systematic deviations in the haptic perception of parallelity. Perception, 28(8), 1001-1012. https://doi.org/10.1068/p2802.

Kappers, A. M. L. (2003). Large systematic deviations in a bimanual parallelity task: Further analysis of contributing factors. Acta Psychologica, 114(2), 131-145. https://doi.org/ 10.1016/S0001-6918(03)00063-5.

Kappers, A. M. L. (2004). The contributions of egocentric and allocentric reference frames in haptic spatial tasks. Acta Psychologica, 117(3), 333-340. https://doi.org/10.1016/j. actpsy.2004.08.002.

Kappers, A. M. L., \& Koenderink, J. J. (1999). Haptic perception of spatial relations. Perception, 28(6), 781-795. https://doi.org/10.1068/p2930.

Kayaert, G., \& Wagemans, J. (2010). Infants and toddlers show enlarged visual sensitivity to nonaccidental compared with metric shape changes. I-Perception, 1, 149-158. https:// doi.org/10.1068/i0397.

Kubilius, J., Sleurs, C., \& Wagemans, J. (2017). Sensitivity to nonaccidental conffgurations of two-line stimuli. I-Perception, 8(2), 2041669517699628.

Kubilius, J., Wagemans, J., \& Op de Beeck, H. P. (2014). Encoding of conffgural regularity in the human visual system. Journal of Vision, 14(9), 11. https://doi.org/10.1167/ 14.9.11.

Lacey, S., Tal, N., Amedi, A., \& Sathian, K. (2009). A putative model of multisensory object representation. Brain Topography, 21(3-4), 269-274. https://doi.org/10.1007/ s10548-009-0087-4.

Landau, B., Gleitman, H., \& Spelke, E. (1981). Spatial knowledge and geometric representation in a child blind from birth. Science (New York, N.Y.), 213(4513), 1275-1278. https://doi.org/10.1126/science.7268438.

Lourenco, S. F., \& Huttenlocher, J. (2008). The representation of geometric cues in infancy. Infancy, 13(2), 103-127. https://doi.org/10.1080/15250000701795572.

Mancini, F., Bolognini, N., Bricolo, E., \& Vallar, G. (2011). Cross-modal processing in the occipito-temporal cortex: A TMS study of the Muller-Lyer illusion. Journal of Cognitive Neuroscience, 23(8), 1987-1997. https://doi.org/10.1162/jocn.2010.21561.

Marmor, G. S., \& Zaback, L.a. (1976). Mental rotation by the blind: Does mental rotation depend on visual imagery? Journal of Experimental Psychology. Human Perception and Performance, 2(4), 515-521. https://doi.org/10.1037/0096-1523.2.4.515.

McGovern, D. P., Astle, A. T., Clavin, S. L., \& Newell, F. N. (2016). Task-speciffc transfer of perceptual learning across sensory modalities. Current Biology. https://doi.org/10.1016/ j.cub.2015.11.048.

Occelli, V., Lacey, S., \& Sathian, K. (2016). Haptic object recognition is view-independent in early blind but not sighted people. https://doi.org/10.1177/0301006615614489.

Overvliet, K. E., Krampe, R. T., \& Wagemans, J. (2012). Perceptual grouping in haptic search: The infiuence of proximity, similarity, and good continuation. Journal of Experimental Psychology: Human Perception and Performance, 38(4), 817. https://doi. org/10.1037/a0029222.

Overvliet, K. E., Krampe, R. T., \& Wagemans, J. (2013). Grouping by proximity in haptic contour detection. PLoS One, 8(6), Article e65412. https://doi.org/10.1371/journal. pone. 0065412 .

Pasqualotto, A., \& Newell, F. N. (2007). The role of visual experience on the representation and updating of novel haptic scenes. Brain and Cognition, 65(2), 184-194. https://doi. org/10.1016/j.bandc.2007.07.009. 
Pasqualotto, A., \& Proulx, M. J. (2012). The role of visual experience for the neural basis of spatial cognition. Neuroscience and Biobehavioral Reviews. https://doi.org/10.1016/j. neubiorev.2012.01.008.

Pasqualotto, A., Spiller, M. J., Jansari, A. S., \& Proulx, M. J. (2013). Visual experience facilitates allocentric spatial representation. Behavioural Brain Research, 236(1), 175-179. https://doi.org/10.1016/j.bbr.2012.08.042.

Peelen, M. V., He, C., Han, Z., Caramazza, A., \& Bi, Y. (2014). Nonvisual and visual object shape representations in occipitotemporal cortex: Evidence from congenitally blind and sighted adults. Journal of Neuroscience, 34(1), 163-170. https://doi.org/10.1523/ JNEUROSCI.1114-13.2014.

Plato. (2005). Plato: Meno and other dialogues. Oxford University Press.

Postma, A., Zuidhoek, S., Noordzij, M. L., \& Kappers, A. M. L. (2007). Differences between early-blind, late-blind, and blindfolded-sighted people in haptic spatial-conffguration learning and resulting memory traces. Perception, 36(8), 1253-1265. https://doi.org/ $10.1068 / \mathrm{p} 5441$.

Postma, A., Zuidhoek, S., Noordzij, M. L., \& Kappers, A. M. L. (2008). Haptic orientation perception beneffts from visual experience: Evidence from early-blind, late-blind, and sighted people. Perception \& Psychophysics, 70(7), 1197-1206. https://doi.org/10.3758/ PP.70.7.1197.

Ptito, M., Matteau, I., Zhi Wang, A., Paulson, O. B., Siebner, H. R., \& Kupers, R. (2012). Crossmodal recruitment of the ventral visual stream in congenital blindness. Neural Plasticity, 2012, 1-9. https://doi.org/10.1155/2012/304045.

Röder, B., Rösler, F., \& Spence, C. (2004). Early vision impairs tactile perception in the blind. Current Biology, 14(2), 121-124. https://doi.org/10.1016/j.cub.2003.12.054.

Ruggiero, G., Ruotolo, F., \& Iachini, T. (2012). Egocentric/allocentric and coordinate/ categorical haptic encoding in blind people. Cognitive Processing, 13(1 SUPPL), 2-6. https://doi.org/10.1007/s10339-012-0504-6.

Schinazi, V. R., Thrash, T., \& Chebat, D. R. (2016). Spatial navigation by congenitally blind individuals. Wiley Interdisciplinary Reviews: Cognitive Science, 7(1), 37-58. https://doi. org/10.1002/wcs.1375.
Serino, A., Giovagnoli, G., de Vignemont, F., \& Haggard, P. (2008). Spatial organisation in passive tactile perception: Is there a tactile ffeld? Acta Psychologica, 128(2), 355-360. https://doi.org/10.1016/j.actpsy.2008.03.013.

Snow, J. C., Strother, L., \& Humphreys, G. W. (2014). Haptic shape processing in visual cortex. Journal of Cognitive Neuroscience, 26(5), 1154-1167. https://doi.org/ 10.1162 /jocn_a_00548.

Spelke, E., Lee, S. A., \& Izard, V. (2010). Beyond core knowledge: Natural geometry. Cognitive Science, 34(5), 863-884. https://doi.org/10.1111/j.15516709.2010.01110.x.

Spelke, E. S., \& Lee, S.a. (2012). Core systems of geometry in animal minds. Philosophical Transactions of the Royal Society, B: Biological Sciences, 367(1603), 2784-2793. https://doi.org/10.1098/rstb.2012.0210.

Tal, N., \& Amedi, A. (2009). Multisensory visual-tactile object related network in humans: Insights gained using a novel crossmodal adaptation approach. Experimental Brain Research, 198(2-3), 165-182. https://doi.org/10.1007/s00221-009-1949-4.

Todd, J. T., Weismantel, E., \& Kallie, C. S. (2014). On the relative detectability of conffgural properties. Journal of Vision, 14(1), 18. https://doi.org/10.1167/14.1.18.

Togoli, I., Crollen, V., Arrighi, R., \& Collignon, O. (2020). The shared numerical representation for action and perception develops independently from vision. Cortex.. https://doi.org/10.1016/j.cortex.2020.05.004.

Van Gool, L. J., Moons, T., Pauwels, E., \& Wagemans, J. (1994). Invariance from the Euclidean geometer's perspective. Perception, 23(5), 547-561. https://doi.org/ $10.1068 / \mathrm{p} 230547$.

Verlaers, K., Wagemans, J., \& Overvliet, K. E. (2015). The effect of perceptual grouping on haptic numerosity perception. Attention, Perception, \& Psychophysics, 77(1), 353-367. https://doi.org/10.3758/s13414-014-0770-8.

Wagemans, J., Lamote, C., \& Van Gool, L. (1997). Shape equivalence under perspective and projective transformations. Psychonomic Bulletin and Review, 4(2), 248-253. https:// doi.org/10.3758/BF03209401. 